\title{
Smac/DIABLO and Cytochrome c Are Released from Mitochondria through a Similar Mechanism during UV-induced Apoptosis
}

\author{
Lingli Zhou ${ }^{1}$, Liying Zhou ${ }^{1}$, Kathy Q. Luo ${ }^{1,2}$ and Donald C. Chang ${ }^{1 *}$ \\ ${ }^{1}$ Department of Biology, The Hong Kong University of Science and Technology, Clear Water Bay, \\ Kowloon, Hong Kong, China; ${ }^{2}$ Bioengineering Graduate Program, Department of Chemical \\ Engineering, The Hong Kong University of Science and Technology, Clear Water Bay, Kowloon, \\ Hong Kong, China
}

During apoptosis, a key event is the release of Smac/DIABLO (an inhibitor of XIAP) and cytochrome c (Cyt-c, an activator of caspase-9) from mitochondria to cytosol. It was not clear, however, whether the releasing mechanisms of these two proteins are the same. Using a combination of single living-cell analysis and immunostaining techniques, we investigated the dynamic process of Smac and Cyt-c release during UV-induced apoptosis in HeLa cells. We found that YFP-labeled Smac and GFP-labeled Cyt-c were released from mitochondria in the same time window, which coincided with the mitochondrial membrane potential depolarization. Furthermore, using immunostaining, we found that the endogenous Smac and Cyt-c were always released together within an individual cell. Finally, when cells were pre-treated with caspase inhibitor (z-VAD-fmk) to block caspase activation, the process of Smac release, like that of Cyt-c, was not affected. This was true for both YFPlabeled Smac and endogenous Smac. These results suggest that in HeLa cells, both Smac and Cyt-c are released from mitochondria during UV-induced apoptosis through the same permeability transition mechanism, which we believe is triggered by the aggregation of $\mathrm{Bax}$ in the outer mitochondrial membrane to form lipid-protein complex.

\section{Introduction}

It is well known that mitochondria play a key role in apoptosis. During the apoptotic process, Cyt-c is released from mitochondria and binds to the adaptor protein Apaf- 1 to activate a caspase cascade. ${ }^{1}$ In addition, in order to neutralize the effect of cytosolic XIAP, which inhibits caspases, a second mitochondrial protein called "Smac/DIABLO" is also released. This protein directly binds to XIAP and removes it from active caspases, and thus allows the caspases to cleave their substrates. ${ }^{2,3}$

It has been well recognized that translocation of these mitochondrial pro-apoptotic proteins (i.e., Cyt-c and Smac) represents an important regulatory mechanism for caspase activation. ${ }^{4}$ So far, it remains elusive whether Cyt-c and Smac are released through the same mechanism or not. Different models had been discussed in the literature. ${ }^{5-8}$ For example, it was reported that the time required for Smac efflux from the mitochondria of HeLa cells subjected to staurosporine (STS)-induced apoptosis was about $19 \pm 3 \mathrm{~min}$, while the time

*Corresponding author:

Running title: Release of Smac and cytochrome c from mitochondria 
required for Cyt-c efflux was $5 \pm 2.5$ min. ${ }^{9}$ Based on this difference, it was suggested that Smac and Cyt-c may exit mitochondria by different pathways. ${ }^{7}$ Recently, Rehm et al. examined the time course of Cyt-c and Smac release during STS-induced apoptosis in MCF-7 cells. ${ }^{6}$ They found that although the average duration of Smac-YFP release (7.8 $\left.\pm 6 \mathrm{~min}\right)$ could be greater than that of Cyt-c-GFP $(2.3 \pm 1.5 \mathrm{~min})$, there was no significant difference in the time to the onset of their release. ${ }^{6}$ This result can be interpreted to suggest that Smac and Cyt-c may be released from mitochondria through the same pathway.

To further clarify this issue, we decided to examine the dynamics of Smac and Cyt-c release in an apoptotic process that is different from that of STS-induced cell death. This study included three parts. First, we used the single living-cell imaging technique to examine the temporal-dependent re-distribution of YFP-labeled Smac and GFP-labeled Cyt-c in living HeLa cells undergoing apoptosis induced by UV-irradiation. A red colored fluorescent dye, Mitotracker, was used to monitor the mitochondrial membrane potential at the same time. Second, we used a double immunostaining method to examine the distribution patterns of endogenous Smac and Cyt-c at different stages of UV-induced apoptosis. A quantitative analysis was performed to determine the correlation between the release of the endogenous Smac and Cyt-c within the same cell. Finally, we studied the dynamics of Smac-YFP release and the distribution patterns of endogenous Smac and Cyt-c with or without the caspase inhibitor. Earlier, we and others had shown that the release of Cyt-c during apoptosis was caspase-independent. ${ }^{9-13}$ Recently, there were conflicting reports on whether Smac release was dependent on the activation of caspase or not. ${ }^{6,8,11,14}$ We would like to examine this point very carefully. If we can demonstrate that the release of Smac, like Cyt-c, is caspaseindependent, it would provide a supporting evidence for the model of co-releasing of Smac and Cyt-c from mitochondria during UV-induced apoptosis.

\section{Materials and Methods}

Chemicals and reagents - Mitotracker Red and goat anti-mouse IgG antibody conjugated with FITC were purchased from Molecular Probes (Eugene, OR); Mouse anti-Cyt-c monoclonal antibody (native) was from PharMingen; Rabbit anti-Cyt-c (H-104) polyclonal antibody (denatured) was from Santa Cruz; Rat anti-Smac monoclonal antibody was from Alexis; Smac-flag plasmid was kindly supplied by Dr. Xiaodong Wang (University of Texas Southwestern Medical Center). Other chemicals were mainly from Sigma.

Cell culture and induction of cell death - HeLa cells were grown in Minimal Essential Medium supplemented with 10\% fetal bovine serum plus $100 \mathrm{U} / \mathrm{ml}$ penicillin and $100 \mu \mathrm{g} / \mathrm{ml}$ streptomycin in $5 \% \mathrm{CO}_{2}$ at $37^{\circ} \mathrm{C}$. Cells were plated on glass coverslips and 24 hrs later cells were exposed to UV-irradiation (UVC at $300 \mu \mathrm{W} / \mathrm{cm}^{2}$ ) for $3 \mathrm{~min}$. When caspase inhibition was needed, cells were pre-treated with z-VAD-fmk $(20 \mu \mathrm{M}) 1 \mathrm{hr}$ before UV-irradiation. The inhibitor was kept in the medium throughout the experimental process.

Generation of the recombinant plasmid pSmac-YFP and gene transfection - Two restriction sites, Bgl II and Hind III were introduced into the 5' and 3' end of the Smac gene by PCR using the following primers: 5'-GGAAGATCTCTGCACAATGGCGGCTCTGAAG AGTTG-3' and 5'-CCCCAAGCTTATCCTCACGCAGGTAGGCC-3'. The resulting PCR product was introduced into the Bgl II and Hind III sites of pEYFP-N1 (clontech). The Cyt-cGFP was kindly supplied by Dr. Brian Herman. ${ }^{15}$ The plasmid was introduced into cells by electroporation. ${ }^{16}$

Imaging methods - Living cell imaging was conducted using a laser scanning confocal microscope (BioRad MRC-600 or Leica DM IRE2). Cells were grown on a coverslip that could fit into an observation chamber. Laser lines of $488 \mathrm{~nm}$ and $568 \mathrm{~nm}$ were used to observe Smac-YFP, Cyt-c-GFP or FITC and Mitotracker Red or Rhodamine, respectively. 
Fluorescent immunostaining — After HeLa cells grown on coverslips were irradiated with UV light, cells were fixed at different time points with $4 \%$ paraformaldehyde plus $0.1 \%$ glutaraldehyde for $15 \mathrm{~min}$ at room temperature, then rinsed 3 times in PBS, permeabilized with $0.2 \%$ Triton X-100 in PBS for 15 min and then blocked in $3 \%$ BSA in PBS at $4{ }^{\circ} \mathrm{C}$ for overnight. After blocking, cells were incubated with mouse anti-native Cyt-c (1:200 dilution in 3\% BSA) and rat anti-Smac (1:100 dilution in 3\% BSA) antibodies at $4{ }^{\circ} \mathrm{C}$ for overnight. Then, cells were stained with secondary antibodies which were goat anti-mouse conjugated with Rhodamine (1:200 dilution) and goat anti-rat conjugated with FITC (1:200 dilution), respectively.

Subcellular fractionation and Western blotting - Cells grown in $60 \mathrm{~mm}$ petri-dishes were collected at the indicated time points and the cytosolic and mitochondrial fractions were separated using a digitonin-based subcellular fractionation technique, basically as described previously. ${ }^{11}$ For Western blotting, proteins (50 $\mu$ g per lane) were analyzed by PAGE using $15 \%$ polyacrylamide gels and followed by electrophoretic transfer onto the nitrocellulose membrane. Then, the membrane was probed (or re-probed) with specific antibodies using the standard procedures.

Measurement of apoptosis - Cells plated on glass coverslips with or without UV treatment were fixed with $4 \%$ paraformaldehyde at the indicated time points. The cells were then stained with Hoechst $33342(0.5 \mu \mathrm{g} / \mathrm{ml})$ for $6 \mathrm{~min}$ at room temperature followed by several washes with PBS. The percentage of cells exhibiting typical apoptotic nuclei, as judged by chromatin condensation and nuclear fragmentation, was assessed by fluorescence microscopy. An average of 1000 - 3000 cells were counted for each sample.

Quantitative analysis of images — We used the MetaMorph v6.0 software (Universal Image, West Chester, PA) to analyze digital images and calculate the mean and SD (standard deviation) of pixel intensities. The release indexes of Smac-YFP and Cyt-c-GFP were determined by measuring the SD of their fluorescence signals within the entire cell. The mitochondrial membrane potential was monitored indirectly by measuring the average intensity of Mitotracker within the cell.

\section{Results}

\section{Smac and Cyt-c were released in a similar time course following the UV treatment based on Western blot analysis}

For purpose of comparison, before we started the single cell analysis, we would like to first compare the time courses of Smac and Cyt-c release in cell populations using a Western-blot analysis. To enhance the Smac signal, we over-expressed Smac-flag in HeLa cells. The presence of Smac and Cyt-c in the cytosolic fractions at different time points after UVirradiation was visualized using anti-Smac and anti-Cyt-c antibodies, respectively. The representative result is shown in Fig. 1. Very little Smac or Cyt-c was detected in the cytosol after one hour of the UV treatment. Both proteins were detected mainly at the 2nd or 3rd hr (Fig. 1A). In this experiment, we used anti-tubulin as a loading control (Fig. 1A). Similar experiments were repeated three times and the quantitative analysis of these results are shown in Fig. 1B. It appears that the cytosolic fraction of Smac and Cyt-c increased in a similar time scale following the UV treatment (Fig. 1B). But, due to a rather large statistical fluctuation, it is difficult to conclude whether both Smac and Cyt-c were released from cells at the same time, or, Smac could be released slightly earlier than that of Cyt-c. The relative large error bars are probably due to two factors. First, the apoptotic process is usually not synchronized between cells. Second, in the Western blot analysis, the intensity of the protein bands may not be linearly proportional to the protein concentrations; it could vary with the 
A

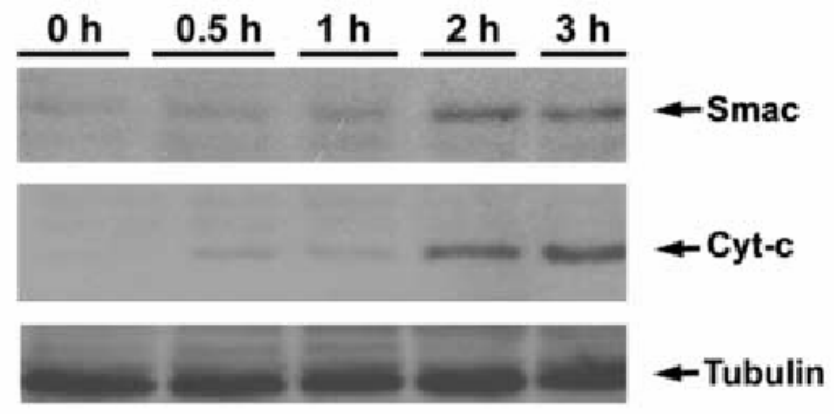

B

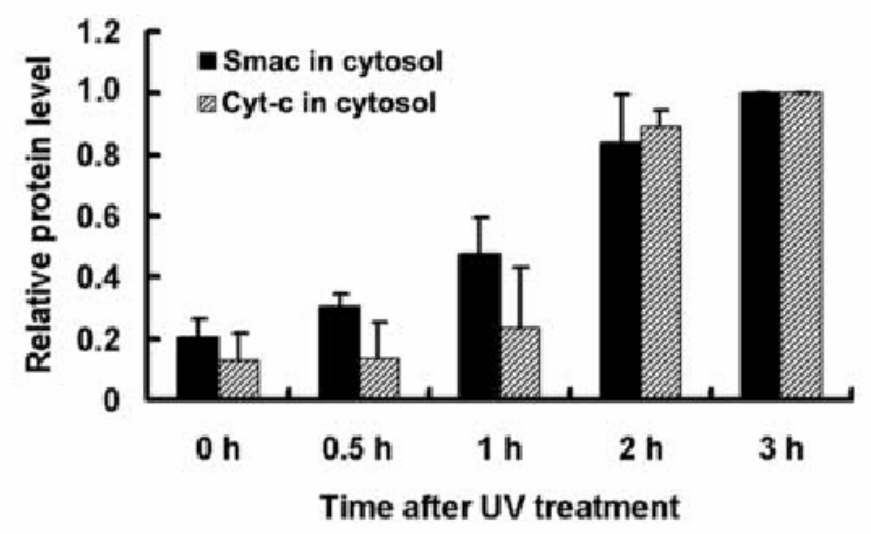

Fig. 1. Western blot analysis of Smac and Cyt-c release during UV-induced apoptosis. (A) Cytosolic fractions of HeLa cells harvested at 0, 0.5, 1, 2 and $3 \mathrm{hr}$ after UV treatment were subjected to 15\% SDS-PAGE analysis. Then, the protein blot was consecutively probed with antibodies against Cyt-c and Smac. The loading control was indicated by the anti-tubulin signal. (B) Quantitative analysis of the Western blot results as shown in (A). The data represented the average of normalized results obtained in three independent experiments.

exposure of the film. Thus, in order to accurately determine the time course of Smac or Cyt-c release, one needs to use a single living-cell analysis.

\section{Single cell measurements indicated that both Smac-YFP and Cyt-c-GFP were released from mitochondria within a narrow time window}

We then investigated the dynamic process of Smac and Cyt-c release in a single living cell during UV-induced apoptosis. We expressed YFP-labeled Smac or GFP-labeled Cyt-c in HeLa cells. The dynamic re-distribution processes of Smac-YFP and Cyt-c-GFP after UVirradiation were recorded using a confocal microscope. Typical results are shown in Fig. 2A and $2 \mathrm{~B}$. It can be seen that, at the early time following the UV treatment, neither Smac nor Cyt-c was released from mitochondria to cytosol; they were distributed in a filamentous pattern that was co-localized with mitochondria, as indicated by the Mitotracker staining (Fig. 2A and 2B). At 150 min after the UV treatment, the cell (marked by an arrow) suddenly started to release Cyt-c from its mitochondria. The fluorescence signal of Cyt-c-GFP appeared to distribute mainly in the cytosol (Fig. 2A). Noted that all mitochondria within the same cell began to release Cyt-c at almost the same time (i.e., in an "all-or-none” manner). A similar dynamic distribution change of Smac-YFP can also be seen in another cell (Fig. 2B). But unlike Cyt-c-GFP, Smac-YFP was not released fully from the mitochondria. We 
estimated that only about half of the mitochondrial Smac-YFP was released into the cytosol; the other half was still retained in the mitochondria. Within about half an hour after the release of Cyt-c-GFP or Smac-YFP, the cell started to shrink and then underwent cell death (Fig. 2A and 2B).

A quantitative analysis of images similar to those shown in Fig. 2A and 2B indicated that the releasing processes of the two mitochondrial proteins were very short. We found that Smac-YFP and Cyt-c-GFP were released from all mitochondria within a cell in a duration of $6.4 \pm 1.7 \mathrm{~min}$ (average of 6 cells) and $5.3 \pm 1.1 \mathrm{~min}$ (average of 5 cells), respectively. Statistically, there was no significant difference between these durations ( $t$ test, $\mathrm{p}=0.193$ ).

A
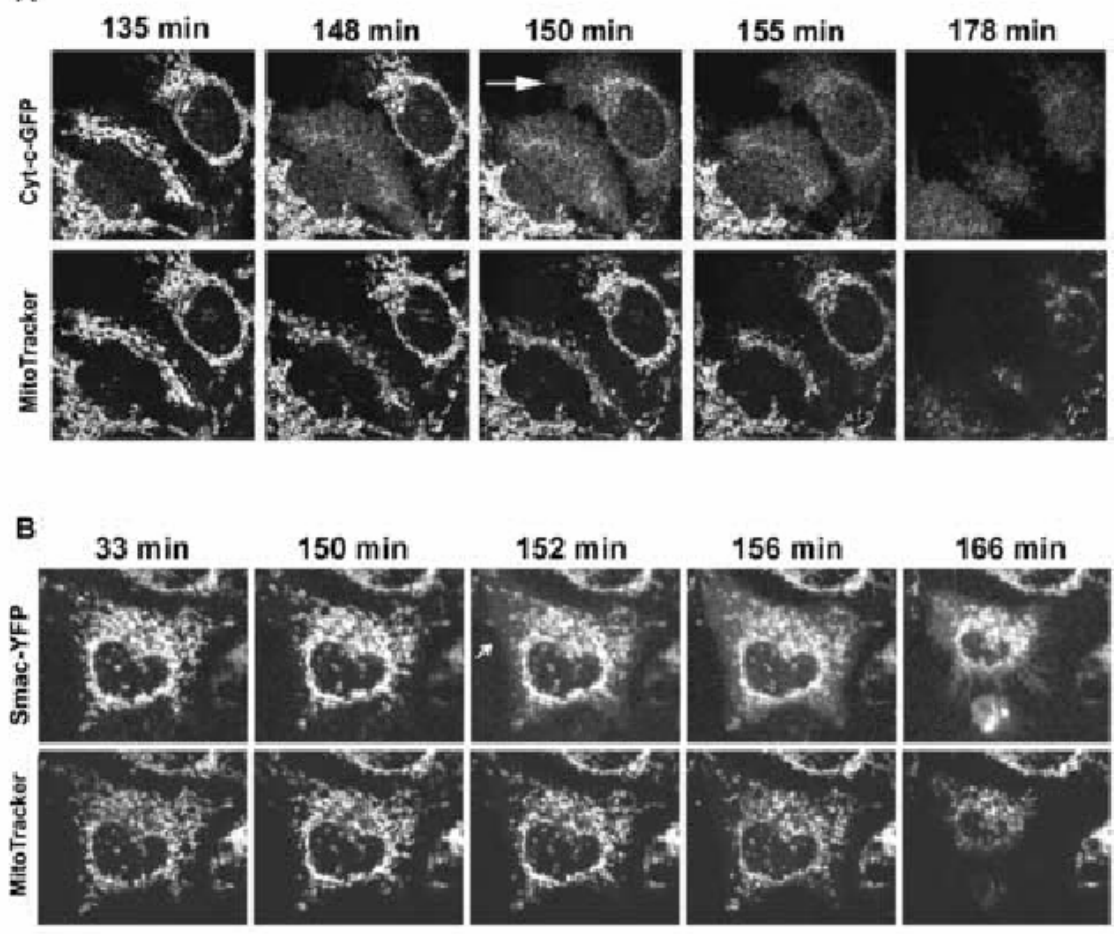

C

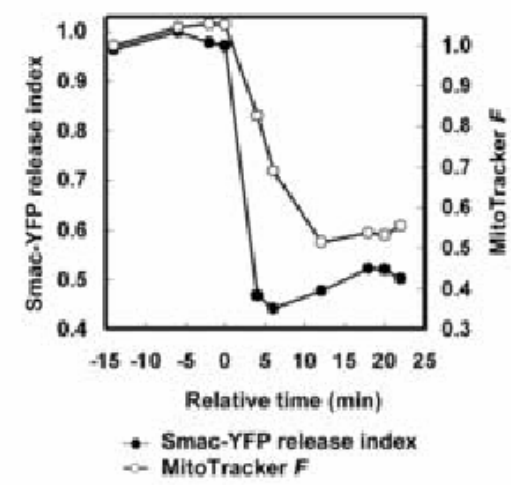

D

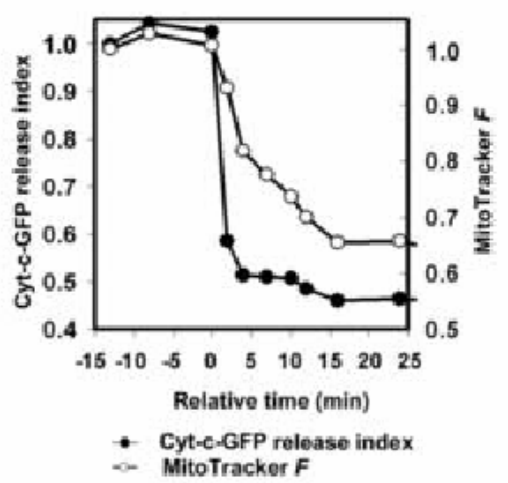

Fig. 2. The dynamics of Smac-YFP and Cyt-c-GFP release during UV-induced apoptosis. (A) Time lapse measurement of Cyt-c-GFP re-distribution dynamics in living HeLa cells. Fluorescent images of Cyt-c-GFP and Mitotracker were recorded by a confocal microscope. The cell beginning to release Cyt-c-GFP from mitochondria to cytosol was indicated by an arrow. (B) Time lapse confocal microscopy measurement of SmacYFP re-distribution in living HeLa cells. The cell beginning to release Smac-YFP from mitochondria to cytosol was marked by an arrow. (C, D) Temporal correlation between Smac-YFP (or Cyt-c-GFP) release and the change of Mitotracker fluorescence signal. The release indexes of Smac-YFP and Cyt-c-GFP were calculated based on single living-cell measurements as shown in panels $\mathbf{A}$ and $\mathbf{B}$. The data were representative of SmacYFP measurements in 6 cells and Cyt-c-GFP measurements in 5 cells. 


\section{The timing of Smac-YFP and Cyt-c-GFP release both coincided with the decrease of mitochondrial membrane potential}

We noticed in these single living-cell measurements that, whenever we observed a cell began to release Smac-YFP (or Cyt-c-GFP) from the mitochondria, the fluorescent signal of Mitotracker was found to decrease in the same cell. Since the fluorescence intensity of Mitotracker is known to be dependent on the mitochondrial membrane potential ( $\Delta \psi_{\mathrm{m}}$ ), we conducted a quantitative examination of the correlation between the release of Smac-YFP or Cyt-c-GFP and the change of $\Delta \psi_{\mathrm{m}}$ during the apoptotic process. Using a confocal microscope operated under the two-channel measurement mode, we imaged Smac-YFP or Cyt-c-GFP and Mitotracker simultaneously in minute intervals after UV treatment. The Smac-YFP and Cyt-c-GFP release indexes were calculated by measuring the standard deviation (SD) of their fluorescence signals in the whole cell using the Metamorph software (see Experimental Procedures). The calculated Smac-YFP and Cyt-c-GFP release indexes, together with the normalized fluorescence intensity of Mitotracker, were plotted over time for each individual cell (Fig. 2C and 2D). A decrease of the fluorescence intensity of Mitotracker was always observed immediately following the Smac-YFP or Cyt-c-GFP releasing process (Fig. 2C and 2D). These results suggested that both Smac-YFP and Cyt-c-GFP were released simultaneously (within minutes) and their release was associated with the reduction of $\Delta \psi_{\mathrm{m}}$ (Fig. 2C and 2D).

\section{The distribution patterns of endogenous Smac and Cyt-c were similar within the same cell}

One potential criticism to the single living-cell measurement is that it depends on monitoring the over-expressed GFP- (or YFP-) labeled proteins instead of the endogenous proteins. For example, since the native Smac usually appears as a tetramer, the YFP-labeled Smac $(\sim 200$ $\mathrm{kDa})$ is significantly larger than the endogenous Smac $(\sim 100 \mathrm{kDa}) \cdot{ }^{2,17}$ We observed that the release of Smac-YFP was not complete. Could this truly reflect the translocation pattern of the endogenous Smac during apoptosis? In order to verify the results obtained from the living-cell analysis, we used a double immunostaining technique to examine simultaneously the distribution patterns of endogenous Smac and Cyt-c following the UV treatment. Representative results are shown in Fig. 3. Before the UV treatment, both Smac and Cyt-c were co-localized in the mitochondria and thus appeared in a filamentous distribution pattern (Fig. 3, panels a-c). After UV treatment, three distribution patterns of Smac and Cyt-c were observed in different cells: (a) Filamentous pattern, which indicated that Cyt-c (or Smac) had not been released from mitochondria (Fig. 3, panel d and e). (b) Diffused pattern, which indicated that Cyt-c (or Smac) had been released completely from mitochondria to cytosol (indicated by arrow in Fig. 3, panel d). (c) Mixed pattern, which indicated that the releasing process was in progress (Fig. 3, panels g-i). In these cells (indicated by arrowhead in Fig. 3, panel g), only part of the Cyt-c (or Smac) was released into the cytosol; a portion of the protein was still retained in the mitochondria.

Results of these immunostaining studies indicated that during UV-induced apoptosis, the endogenous Smac could be completely released from mitochondria to cytosol (Fig. 3, panel d). The partial release of Smac-YFP observed in the living cell measurements was probably due to a protein maturation process related to over-expression of the YFP-labeled Smac (For details, see Discussion).

A quantitative analysis of our double immunostaining experiments strongly indicated that the endogenous Smac and Cyt-c were co-released from mitochondria during UV-induced apoptosis (Table 1). If the release of Smac and Cyt-c were independent, we would expect that their distribution patterns would not be correlated with each other. But as shown in Table 1, 


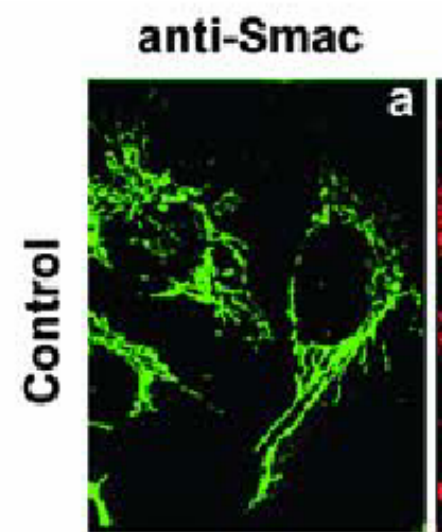

anti-Cyt-c
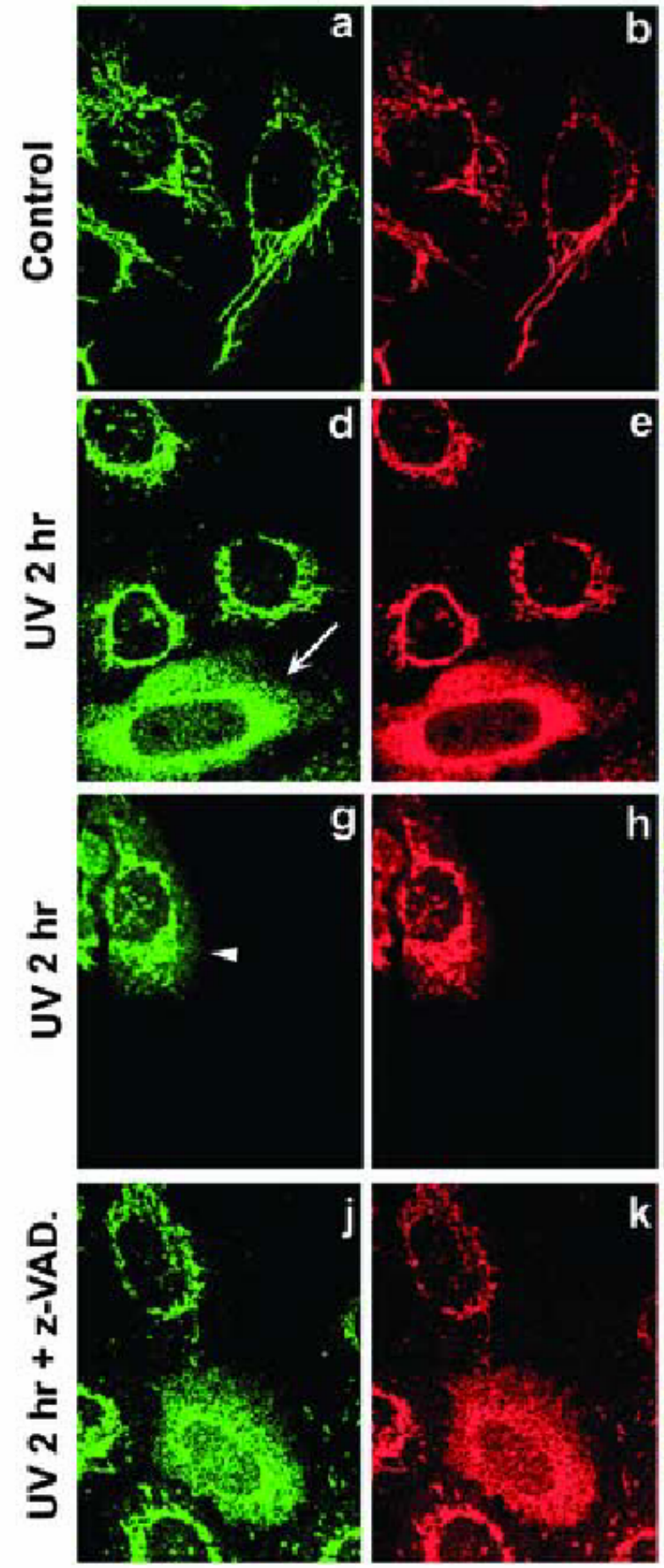
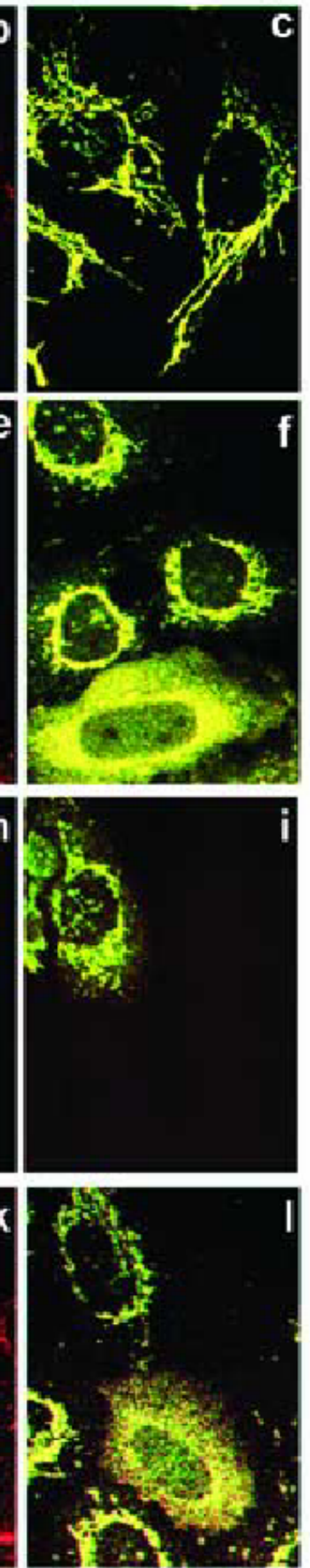

Fig. 3. Distribution of endogenous Smac and Cyt-c in HeLa cells during UV-induced apoptosis. HeLa cells were fixed at different times following UV treatment and were double stained with antibodies against Smac (green) and Cyt-c (red). The merged images are shown in the right column. (Panel a-c) Before UVtreatment, both Smac and Cyt-c were co-localized with the mitochondria and appeared as filamentous structures. (Panel d-i) 2 hrs after HeLa cells were UV-irradiated. Note that in one cell (marked by an arrow in panel d), both Cyt-c and Smac were released from mitochondria and appeared in a diffused pattern throughout the cell. In another cell (marked by an arrowhead in panel g), a partially released pattern of Smac and Cyt-c could be seen. (Panel j-l) HeLa cells pre-incubated with z-VAD-fmk $(20 \mu \mathrm{M})$ were fixed at 2 hrs after UV-irradiation. Note that in the lower cell, both Smac and Cyt-c were distributed in a diffused pattern. 
the distribution patterns of Smac and Cyt-c were highly correlated. Whenever Smac was observed to have a particular distribution pattern (say, diffused) in a cell, we would found an identical distribution pattern of Cyt-c (i.e., diffused) in the same cell. Over the thousands of UV-treated cells that we had analyzed, we did not find even a single cell in which the endogenous Smac was distributed in a diffused pattern, while Cyt-c was in a filamentous pattern (or vise versa). The strong correlation between the distribution patterns of Smac and Cyt-c can be easily seen in Table 1; almost all cells were classified in the diagonal areas of the table. This demonstrated that the distribution patterns of Smac and Cyt-c were mostly identical in a UV-treated cell, suggesting that Smac and Cyt-c must be released by a similar process during apoptosis.

Table 1 Number of cells showing different types of morphological distribution of endogenous Cyt-c and Smac

\begin{tabular}{|c|c|c|c|c|c|}
\hline & & Filamentous & Mixed & Diffused & $\begin{array}{c}\text { Total number } \\
\text { of cells analyzed }\end{array}$ \\
\hline \multirow{3}{*}{ Control } & Filamentous & 2482 & 0 & 0 & \multirow{3}{*}{2507} \\
\hline & Mixed & 0 & 18 & 0 & \\
\hline & Diffused & 0 & 0 & 7 & \\
\hline \multirow{3}{*}{$\begin{array}{c}\text { UV } \\
(2 \mathrm{hr})\end{array}$} & Filamentous & 1546 & 1 & 0 & \multirow{3}{*}{2939} \\
\hline & Mixed & 2 & 314 & 0 & \\
\hline & Diffused & 0 & 1 & 1075 & \\
\hline \multirow{3}{*}{$\begin{array}{c}\text { UV } \\
(4 \mathrm{hr})\end{array}$} & Filamentous & 5 & 0 & 0 & \multirow{3}{*}{643} \\
\hline & Mixed & 0 & 16 & 0 & \\
\hline & Diffused & 0 & 0 & 622 & \\
\hline
\end{tabular}

\section{Smac release during $\mathrm{UV}$-induced apoptosis was independent of caspase activation}

It had been shown previously by us and others that Cyt-c release from mitochondria during UV-induced apoptosis is not dependent on the activation of caspase. ${ }^{9-12}$ Whether Smac release is caspase-dependent or not was not yet clear. Several earlier studies reported that the release of Smac appears to require caspase activity during apoptosis induced by TRAIL or other stimuli, ${ }^{8,11}$ implying that cells may use a mechanism different from that of Cyt-c to release Smac. To examine this point directly, we studied the effect of a caspase inhibitor, zVAD-fmk, on Smac release during UV-induced apoptosis. HeLa cells were pretreated with zVAD-fmk $(20 \mu \mathrm{M})$ for $1 \mathrm{hr}$ prior to UV treatment. The numbers of apoptotic cells were determined based on Hoechst staining of the nucleus. We found that although the z-VADfmk treatment could prevent cell death (Fig. 4A), it had no significant effect on the release of Smac-YFP (Fig. 4B). These results suggest that Smac release is not dependent on caspase activation.

To further demonstrate this point, we used single living-cell imaging to study the dynamic redistribution of Smac-YFP during UV-induced apoptosis with or without z-VADfmk treatment. In the absence of $\mathrm{z}$-VAD-fmk, cells were observed to undergo cell shrinkage and cell death within half an hour following the release of Smac-YFP (Fig. 4C). In the presence of z-VAD-fmk, the UV-treated cells could also release Smac-YFP from mitochondria (indicated by arrow in Fig. 4C). But, the cell did not die even at 2 hrs after Smac- YFP was released. Thus, it appears that the caspase inhibitor can only affect events downstream of Smac release. 
A

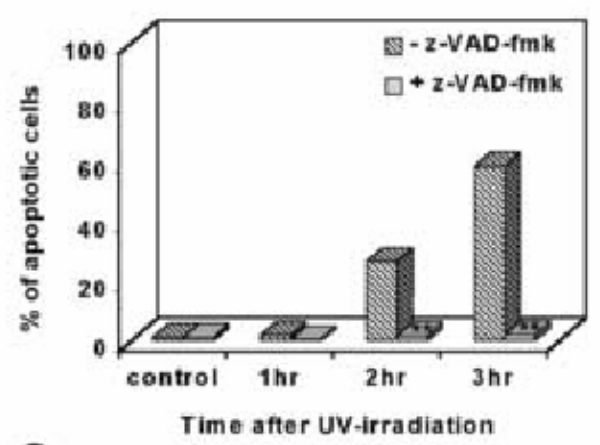

C

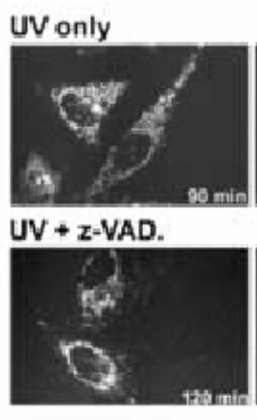

D

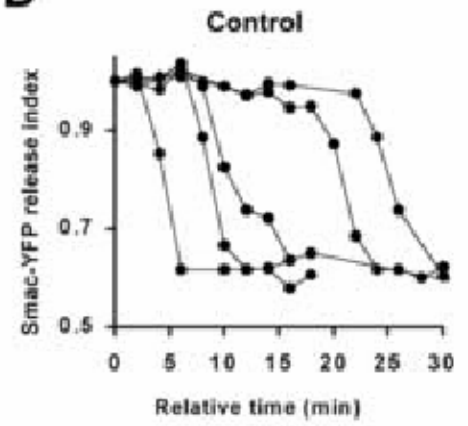

B

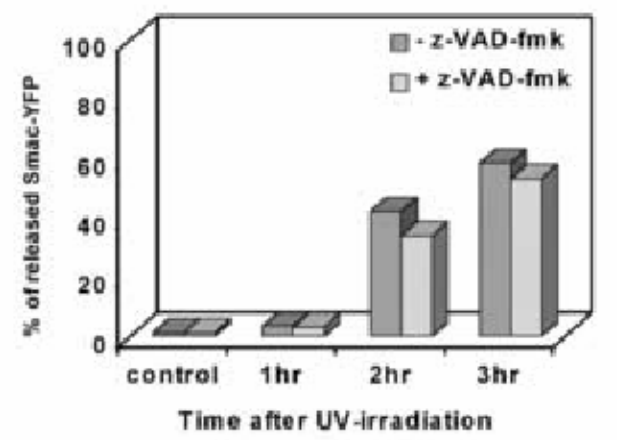

Time after UV-irradiation
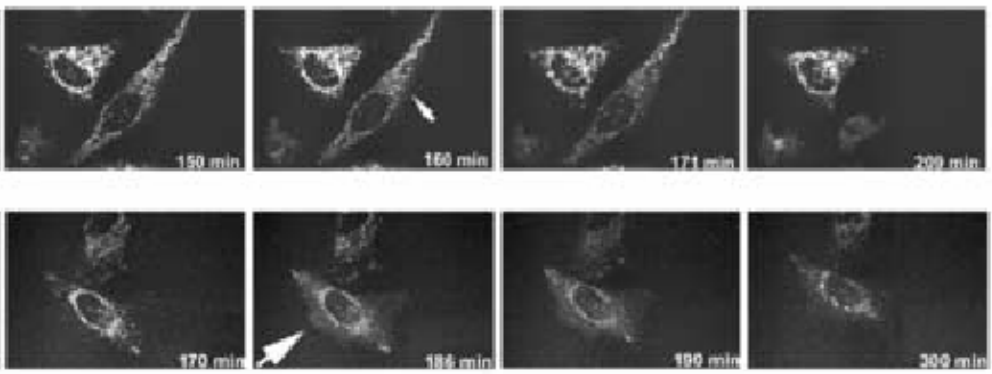

E

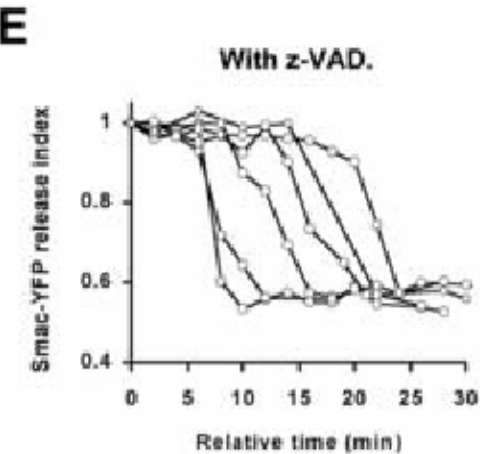

Fig. 4. Effects of the caspase inhibitor $z-V A D-f m k$ on $U V$-induced apoptosis and release of Smac. (A, B) HeLa cells with or without z-VAD-fmk treatment were assayed for UV-induced apoptosis or Smac release. The percentage of apoptotic cells was determined by DNA staining. The percentage of cells releasing Smac-YFP was determined by fluorescence microscopy. The comparison between two groups was performed by chi-square test. ** means $p<0.001$. (C) Sample records of time-lapse confocal measurements of Smac-YFP re-distribution during UV-induced apoptosis with and without z-VAD-fmk $(20 \mu \mathrm{M})$. (D, E) The dynamics of Smac-YFP release in various individual cells during UV-induced apoptosis with (E) or without (D) z-VAD-fmk treatment.

Using single living-cell analysis, we had compared the dynamics of Smac-YFP release during UV-induced apoptosis of six z-VAD-fmk pretreated cells with that of five control cells. The results are showed in Fig. $4 \mathrm{E}$ and $4 \mathrm{D}$. The time courses in both cases were very similar. Also, there was no significant difference of the duration of Smac-YFP release between the two groups ( $6.4 \pm 1.7$ min vs $6.5 \pm 2.2 \mathrm{~min}, \mathrm{p}=0.935$ ).

Finally, using the immunostaining method, we examined the distribution patterns of both Smac and Cyt-c in UV-irradiated HeLa cells pretreated with z-VAD-fmk. We observed that in many of the z-VAD-fmk treated cells, both Smac and Cyt-c had a diffused distribution pattern (Fig. 3, panel j-l). This finding indicated that Smac was co-released with Cyt-c from mitochondria regardless whether caspase activation was inhibited or not. Indeed, our 
quantitative analysis of the distribution patterns of endogenous Smac and Cyt-c in UV-treated cells indicated that, in the presence of z-VAD-fmk, Cyt-c and Smac were always co-released in the same cell (Table 2).

Table 2 Number of cells showing different types of morphological distribution of Cyt-c and Smac in the presence of z-VAD-fmk

\begin{tabular}{|c|c|c|c|c|c|}
\hline & Cyt- & Filamentous & Mixed & Diffused & $\begin{array}{c}\text { Total number } \\
\text { of cells analyzed }\end{array}$ \\
\hline \multirow{3}{*}{$\begin{array}{l}\text { UV } \\
(1 \mathrm{hr})\end{array}$} & Filamentous & 827 & 0 & 0 & \multirow{3}{*}{1103} \\
\hline & Mixed & 0 & 109 & 0 & \\
\hline & Diffused & 0 & 0 & 167 & \\
\hline \multirow{3}{*}{$\begin{array}{l}\text { UV } \\
(2 \mathrm{hr})\end{array}$} & Filamentous & 32 & 0 & 0 & \multirow{3}{*}{845} \\
\hline & Mixed & 0 & 149 & 0 & \\
\hline & Diffused & 0 & 9 & 655 & \\
\hline \multirow{3}{*}{$\begin{array}{l}\text { UV } \\
(4 \mathrm{hr})\end{array}$} & Filamentous & 9 & 0 & 0 & \multirow{3}{*}{657} \\
\hline & Mixed & 0 & 107 & 0 & \\
\hline & Diffused & 0 & 0 & 541 & \\
\hline
\end{tabular}

\section{Discussion}

Since Cyt-c and Smac/DIABLO are two key regulators of the caspase system in apoptosis, recently there has been a strong interest in studying their releasing mechanisms. ${ }^{5-8,11,18-21}$ One major question is whether these two mitochondrial proteins are released through the same mechanism or not. So far, there has been a diverse of ideas on this point. Several studies suggested that the releasing mechanisms for Smac and Cyt-c may be different. ${ }^{7,8,11}$ Results from other studies, however, suggested that the releasing mechanisms for Smac and Cyt-c might be the same. ${ }^{5,6}$ Part of this difference could be related to the different cell models used in the previous studies, and part of it could be due to the difficulty of correlating the transport properties of two proteins in a cell population. As we have demonstrated in this study, it is rather difficult to conclude from the Western blot analysis about the differential time profiles of proteins that are released in a roughly similar time scale. To overcome these problems, we used a combination of single living-cell analysis and immunostaining techniques to investigate the dynamic process of Smac and Cyt-c release during UV-induced apoptosis in HeLa cells. We found that YFP-labeled Smac and GFP-labeled Cyt-c were released from mitochondria in the same time window, which coincided with the beginning of depolarization of the mitochondrial membrane potential. Furthermore, based on an immunostaining study, we found that the endogenous Smac and Cyt-c were always released together within the same cell. Finally, when cells were pre-treated with caspase inhibitor (z-VAD-fmk) to block caspase activation, the process of Smac release, like that of Cyt-c, was not affected. This was true for both YFP-labeled Smac and endogenous Smac. These results strongly suggest that, in 
HeLa cells, both Smac and Cyt-c are released from mitochondria during UV-induced apoptosis through the same permeability transition mechanism, which is upstream of caspase9 and caspase- 3 activation.

Earlier, Rehm et al. had utilized a single cell analysis method to monitor the temporal relationship between releases of YFP-labeled Smac and GFP-labeled Cyt-c in STS-induced apoptosis in MCF-7 cells. ${ }^{6}$ Although they did not examine whether the distribution of endogenous Smac was truly reflected by that of YFP-labeled Smac, their conclusion nevertheless was consistent with our findings here. This agreement may suggest that the conclusion of a common releasing mechanism for Smac and Cyt-c is rather general and is not limited to UV- or STS-induced apoptosis. Of course, to really prove this point, one will have to further examine the release dynamics of Smac and Cyt-c in other major apoptotic pathways, such as those induced by TRAIL or oxidative stress.
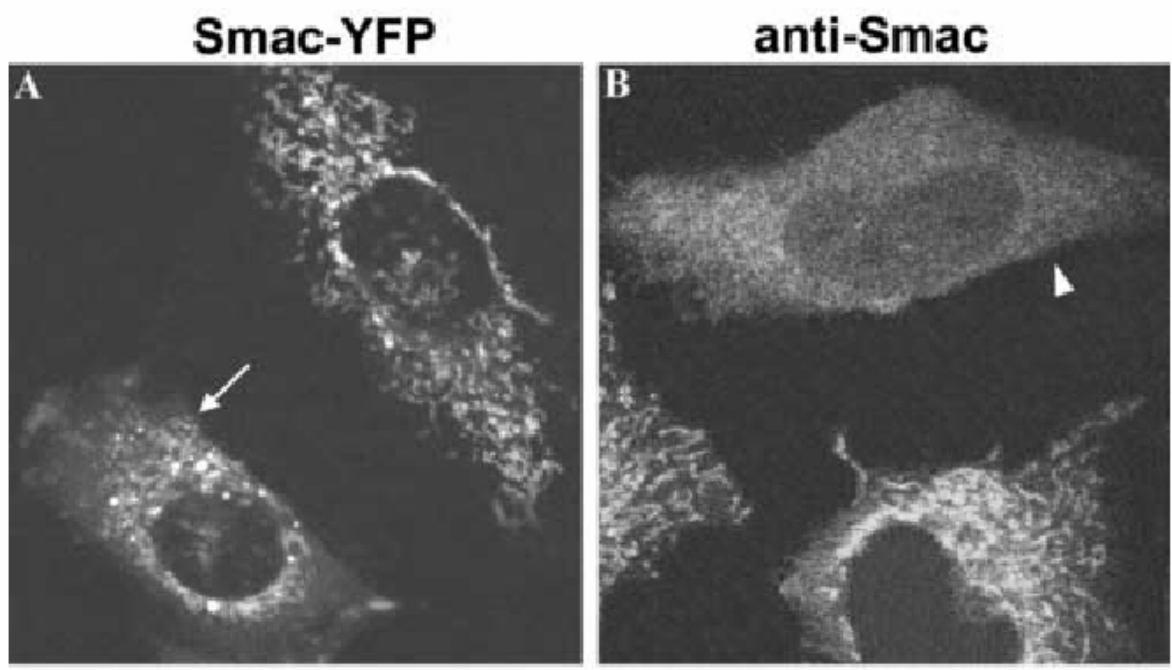

Fig. 5. Comparing the releasing patterns of Smac-YFP and endogenous Smac in HeLa cells during UVinduced apoptosis. (A) HeLa cells were transfected with Smac-YFP and treated with UV. Only part of the Smac-YFP was released from mitochondria during apoptosis (See the cell marked by arrow). (B) Immunostaining of HeLa cells showing the distribution pattern of endogenous Smac at 2 hrs after UV treatment. In the cell undergoing apoptosis, Smac was fully released from mitochondria (See the cell marked by arrow head).

Results of this study may shed some light on the molecular nature of the releasing mechanism of Cyt-c and Smac. So far, several hypotheses have been proposed for the release of mitochondrial proteins during apoptosis, ${ }^{22,23}$ including: (a) Swelling of mitochondrial matrix and rupture of the outer mitochondrial membrane as a result of PT pore opening; ${ }^{24}$ (b) Formation of a novel pore by the Bax family proteins in conjunction with the voltage dependent anion channel (VDAC); ${ }^{19}$ (c) Formation of channels comprised of Bax family members; ${ }^{25}$ (d) Formation of large lipid-protein complexes in the mitochondrial outer membrane comprised mainly of Bax-like proteins. ${ }^{22,26}$ The swelling model (i.e., hypothesis a) has already been ruled out by our previous study. ${ }^{10}$ In view of the results of this study, we think that models based on hypotheses $\mathbf{b}$ and $\mathbf{c}$ may also be discarded. These two models assume that the mitochondrial proteins were released through a "channel" formed by oligomer of Bax family proteins. It is conceivable that such channel may be large enough to pass a small molecule such as Cyt-c (MW=15 kDa). ${ }^{25}$ But, the channel would be very difficult to allow Smac to pass through. It is known that the endogenous Smac generally appeared as tetramers in the cellular environment, its molecular weight is about $100 \mathrm{kDa}{ }^{2}$ For the YFP-labeled Smac, it would have even a larger size $(\sim 200 \mathrm{kDa})$. From crystal 
structure studies, it was suggested that Smac may form dimers instead of tetramers. ${ }^{17}$ But even if the dimer model is true in the in vivo environment, the molecular weight of the YFPSmac dimer would still approach $100 \mathrm{kDa}$. It is impossible for such a large protein complex to pass through a channel. Hence, we believe only the model based on hypothesis $\mathbf{d}$ could explain our findings. Recently, Kuwana et al. reported that Bax could produce mega openings at the mitochondrial outer membrane, which could release supramolecules up to $2000 \mathrm{kDa} .^{27}$ With such a model, one can expect that Smac and Cyt-c are released together upon the aggregation of Bax in the outer mitochondrial membrane.

In the living cell measurement, we observed that the release of Smac-YFP from mitochondria during apoptosis was not complete. Similar observation was also reported previously. ${ }^{7}$ Our immunostaining study, however, indicated that the endogenous Smac was released fully from mitochondria (See Fig. 5). How can one explain the difference between the behaviour of Smac-YFP and the endogenous Smac? At the beginning, we suspected that it might be due to the larger molecular size of Smac-YFP in comparison to the endogenous Smac. Thus, we did a comparative study on the release of Smac-flag, Smac-YFP and endogenous Smac based on Western-blot (results not shown). We found that Smac-flag, whose size was close to the endogenous Smac, also showed a partial release pattern. Hence, we concluded that the partial release of Smac-YFP was not due to its large molecular size, instead, it was probably due to gene over-expression. In general, most proteins destined for the intermembrane space are initially synthesized as larger precursor polypeptides carrying two signal sequences at $\mathrm{N}$-terminal, a matrix-targeting signal followed by an intermembrane space-sorting signal. ${ }^{28,29}$ After the protein is imported into the mitochondrial matrix, its matrix-targeting signal is cleaved by the mitochondrial processing peptidase. Then, the intermembrane space-sorting signal is removed by the inner membrane peptidase, and the mature polypeptide is released into the intermembrane space. ${ }^{29}$ Smac/DIABLO is synthesized as a 239 amino acid precursor protein, with the $\mathrm{NH}_{2}$-terminal 55 amino acids serving as a mitochondrial targeting sequence. ${ }^{2,3}$ We suspected that the precursor Smac, like most mitochondrial proteins, is accumulated in the mitochondrial matrix first. Later, its N-terminus is removed by proteolysis to generate the mature form of Smac, which subsequently enters the mitochondrial inter-membrane space. Since the enzyme for cleaving the mitochondrial localization signal is limited, when a large amount of Smac-YFP is over-expressed, a certain portion of the full-length Smac-YFP molecules may still be waiting for processing in the matrix. As a result, only the mature form of Smac-YFP in the inter-membrane space can be released from mitochondria to cytosol during apoptosis. This hypothesis can be tested in the future using EM techniques.

\section{Acknowledgements}

We thank Drs. Yongmei Pu and Naihan Xu and Ms. Vivian Yu for technical assistance. The Smac-flag and Cytc-GFP plasmids were kindly supplied by Dr. Xiaodong Wang and Dr. Brian Herman, respectively. This work was supported by the Research Grants Council of Hong Kong (HKUST6109/01M HKUST6104/02M, and AoE) and the EHIA project of HKUST.

\section{References:}

1. Finkel E. The mitochondrion: is it central to apoptosis? Science 2001; 292: 624-626.

2. Du C, Fang M, Li Y, Li L, Wang X. Smac, a mitochondrial protein that promotes cytochrome c-dependent caspase activation by eliminating IAP inhibition. Cell 2000; 102: 33-42.

3. Verhagen AM et al. Identification of DIABLO, a mammalian protein that promotes apoptosis by binding to and antagonizing IAP proteins. Cell 2000; 102: 43-53.

4. Wang X. The expanding role of mitochondria in apoptosis. Genes Dev 2001; 15: 2922-2933.

5. Madesh M, Antonsson B, Srinivasula SM, Alnemri ES, Hajnoczky G. Rapid kinetics of tBid-induced cytochrome c and Smac/DIABLO release and mitochondrial depolarization. J Biol Chem 2002; 277: 56515659. 
6. Rehm M, Dussmann H, Prehn JH. Real-time single cell analysis of Smac/DIABLO release during apoptosis. J Cell Biol 2003; 162: 1031-1043.

7. Springs SL, Diavolitsis VM, Goodhouse J, McLendon GL. The kinetics of translocation of Smac/DIABLO from the mitochondria to the cytosol in HeLa cells. J Biol Chem 2002; 277: 45715-45718.

8. MacFarlane M, Merrison W, Bratton SB, Cohen GM. Proteasome-mediated degradation of Smac during apoptosis: XIAP promotes Smac ubiquitination in vitro. J Biol Chem 2002; 277: 36611-36616.

9. Goldstein JC, Waterhouse NJ, Juin P, Evan GI, Green DR. The coordinate release of cytochrome c during apoptosis is rapid, complete and kinetically invariant. Nat Cell Biol 2000; 2: 156-162.

10. Gao W, Pu Y, Luo KQ, Chang DC. Temporal relationship between cytochrome c release and mitochondrial swelling during UV-induced apoptosis in living HeLa cells. J Cell Sci 2001; 114: 2855-2862.

11. Adrain C, Creagh EM, Martin SJ. Apoptosis-associated release of Smac/DIABLO from mitochondria requires active caspases and is blocked by Bcl-2. Embo $J$ 2001; 20: 6627-6636.

12. Waterhouse NJ et al. Cytochrome c maintains mitochondrial transmembrane potential and ATP generation after outer mitochondrial membrane permeabilization during the apoptotic process. J Cell Biol 2001; 153: 319-328.

13. Finucane DM, Bossy-Wetzel E, Waterhouse NJ, Cotter TG, Green DR. Bax-induced caspase activation and apoptosis via cytochrome c release from mitochondria is inhibitable by Bcl-xL. J Biol Chem 1999; 274: 2225-2233.

14. Arnoult D et al. Mitochondrial release of AIF and EndoG requires caspase activation downstream of Bax/Bak-mediated permeabilization. Embo J 2003; 22: 4385-4399.

15. Mahajan NP et al. Bcl-2 and Bax interactions in mitochondria probed with green fluorescent protein and fluorescence resonance energy transfer. Nat Biotechnol 1998; 16: 547-552.

16. Chang DC. Experimental strategies in efficient transfection of mammalian cells. Electroporation. Methods Mol Biol 1997; 62: 307-318.

17. Chai J et al. Structural and biochemical basis of apoptotic activation by Smac/DIABLO. Nature 2000; 406: 855-862.

18. Carson JP et al. Smac is required for cytochrome c-induced apoptosis in prostate cancer LNCaP cells. Cancer Res 2002; 62: 18-23.

19. Shimizu S, Narita M, Tsujimoto Y. Bcl-2 family proteins regulate the release of apoptogenic cytochrome c by the mitochondrial channel VDAC. Nature 1999; 399: 483-487.

20. Sun XM, Bratton SB, Butterworth M, MacFarlane M, Cohen GM. Bcl-2 and Bcl-xL inhibit CD95-mediated apoptosis by preventing mitochondrial release of Smac/DIABLO and subsequent inactivation of X- linked inhibitor-of-apoptosis protein. J Biol Chem 2002; 277: 11345-11351.

21. Usuda $\mathrm{J}$ et al. Promotion of photodynamic therapy-induced apoptosis by the mitochondrial protein Smac/DIABLO: dependence on Bax. Photochem Photobiol 2002; 76: 217-223.

22. Desagher S, Martinou JC. Mitochondria as the central control point of apoptosis. Trends Cell Biol 2000; 10: 369-377.

23. van Loo G et al. The role of mitochondrial factors in apoptosis: a Russian roulette with more than one bullet. Cell Death Differ 2002; 9: 1031-1042.

24. Crompton M. The mitochondrial permeability transition pore and its role in cell death. Biochem J $1999 ; 341$ ( Pt 2): 233-249.

25. Saito M, Korsmeyer SJ, Schlesinger PH. BAX-dependent transport of cytochrome c reconstituted in pure liposomes. Nat Cell Biol 2000; 2: 553-555.

26. Basanez $\mathrm{G}$ et al. Bax, but not Bcl-xL, decreases the lifetime of planar phospholipid bilayer membranes at subnanomolar concentrations. Proc Natl Acad Sci U S A 1999; 96: 5492-5497.

27. Kuwana $\mathrm{T}$ et al. Bid, Bax, and lipids cooperate to form supramolecular openings in the outer mitochondrial membrane. Cell 2002; 111: 331-342.

28. Gakh O, Cavadini P, Isaya G. Mitochondrial processing peptidases. Biochim Biophys Acta 2002; 1592: 6377.

29. Neupert W. Protein import into mitochondria. Annu Rev Biochem 1997; 66: 863-917.

\section{Footnotes}

The abbreviations used are: Smac/DIABLO, second mitochondria-derived activator of caspase/direct inhibitor of apoptosis (IAP)-binding protein with low pI; XIAP, X-chromosome linked inhibitor of apoptosis; Cyt-c, cytochrome c; GFP, Green Fluorescence Protein; YFP, Yellow Fluorescence Protein; z-VAD-fmk, Nbenzyloxycarbonyl-Val-Ala-Asp-fluoromethylketone; VDAC, voltage dependent anion channel; Apaf-1, apoptotic protease-activating factor-1; UV, ultra-violet light. 